\title{
Design and Performance Analysis of a New Framework of Multipath Load Balancing Routing in Ad-Hoc Network
}

\author{
K. R. Yadav, Dinesh Goyal, Narendra Yadav, Devesh Kr. Bandil
}

\begin{abstract}
In the communication networks, one of the major problems in the quality of service is congestion. Indeed, the phenomenon of congestion is due to the fact that many packets emitted follow the same paths. As a result, the increase the bandwidth offered can solve the problem only in the short term. In order to solve this problem, several solutions have been proposed, such as multipath routing algorithms, which distribute traffic between a source and a destination on several different paths, while taking into account the level of saturation of the intermediaries nodes, composing the different paths with a objective to avoid cluttered paths. Its purpose is to shed the excessive burden save a knot on its neighbors to improve performance and to exploit efficiently. In this paper we have analysis the load balancing in Multipath routing to achieve better network performance. We have study and reviewed the different load balancing algorithm in multipath network and based on review we have proposed a new load balancing algorithm in the multipath network. We have modified the packet of RREP and $R R E Q$ packet to transmit and receive the route information to compute the load of every path and we added a new field in RREP packet Buffer size to store the current node and their neighbor information to find the alternate route if network load in increase we have compare the performance of proposed protocol with the LB-AOMDV, SMR \& AOMDV multipath routing protocols. NS2 is used for simulation.
\end{abstract} RREQ

Keyword: Load Balancing, Multipath, Routing, RREP,

\section{INTRODUCTION}

The rapid evolution of mobile node processing capabilities and the improvement of the quality of the network linking these entities motivated the use of the ad hoc network as support for distributed calculations. A good use of this platform allows increasing the potential power of the nodes. The need for efficient load sharing and balancing algorithm is graver when the environment of network is more heterogeneous. Indeed, mobile computers have intrinsic resources (computing power, battery power) very variable peace rates, which may vary from resources comparable to those of PCs, in the case of laptops, to significantly lower resources, especially in the case of handheld computers.

Revised Manuscript Received on November 25, 2019.

* Correspondence Author

K. R. Yadav Research Scholar, Department of Computer Science \& Engineering, SGVU, Jaipur

Dinesh Goyal, Professor, Department of CSE, Poornima Institute of Engineering \& Technology, Jaipur

Narendra Yadav, Associate Professor, Department of CSE, Manipal University, Jaipur

Devesh Kr. Bandil, Professor, Suresh Gyan Vihar University, Jaipur
This processing capacity heterogeneous can cause situations where nodes are heavily loaded, while others remain poorly charged or unused. Proper use of each node helps to take advantage of its power without affecting its own work and potentially increase the power of the mobile nodes.

Ad-Hoc network consists of a collection of wireless mobile nodes, which constitute a temporary network without depending on an existing infrastructure or centralized administration [1]. Nowadays, people are moving and communicating more and more. They need new technologies that allow them to recover quickly and easily share information and communicate with distant people who may not be door where in the world. Recent years have seen the technological development of numerous components and electronic devices of all kinds to respond to these new needs. These communicating devices are becoming smaller, have capacities more and more efficient computing and are becoming more and more widespread. We meet them everywhere in our daily lives: at home, at the office, in cars, etc. Advancing research in the field of mobile networks and technologies communication has favored the design of new applications (audio and video) conference, television on demand, interactive group games, tele-teaching. . .etc) and the formation of collaborative groups. Several of these applications, such as communications of groups or the coordination of civil forces deploy on ad-hoc networks and require effective information shifts to increase collective knowledge. The most rudimentary modes of collaboration are limited to simple exchanges text messages (forums, mailing lists). However, users are becoming more aspirants for solutions that allow them to collaborate in situations of mobility with other users in order to offer their applications features that allow them to run faster and work to reduce their consumption energy. These new needs create new challenges to ensure group communications in an ad hoc network, offering quality of service for applications (reduced response time), while adapting to the nature of networks. Wireless Networks \& their Ad hoc characteristics, mobility and dynamism of the nodes, energy. However, these mobile environments have a significant heterogeneous great variability both in terms of the processing capacity and the energy between the different nodes. Indeed, a more powerful node in terms of processing capacity can to become idle, because he has quickly finished his work while the others, less powerful, are occupied for most of the time and thus consume more energy. The capacity of the powerful nodes can be exploited by overcrowded nodes if a fraction the charge of these is shared with them. The use of load balancing as solution for distribution of load is therefore crucial for improving the

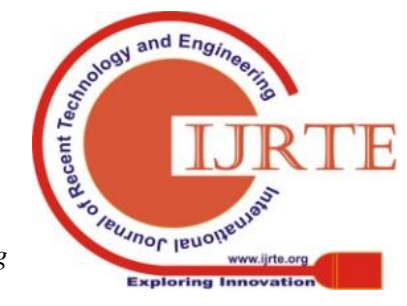


performance of an ad hoc network and satisfies the services and applications of mobile users. Moreover, despite the technological progress made, the energy is still a problem. In an ad hoc environment, given that mobile hosts are equipped with powerful batteries whose capacity of Consumption of energy in mobile hosts is a challenge for the duration of life of the network; as soon as the energy is dissipated at certain nodes may result in network partitioning and loss of connection as a result.

Thus, for an ad hoc network to have maximum longevity and to maintain the network functional as long as possible, protocols designed for ad hoc networks must use the limited energy resources wisely. All of these challenges must be addressed by taking into account the intrinsic constraints of technological entities and communication technologies. We present in this work a new load balancing algorithm where the idea the basic thing is to transfer the load between the nodes while avoiding the inactivity of the nodes. Those tasks remain pending on other nodes. We are interested in two important changes: the computing load, related to the services and applications supported by each node, and the communication load. A load balancing strategy is considered effective if it generates a low overhead of communication due to energy and bandwidth limited in ad hoc networks. Our contribution includes mechanisms for load balancing in a ad-hoc network. Energy management in ad hoc networks is crucial as the nodes have severe energy constraints. In fact, the limited energetic capacity of the nodes imposes the communication mode within these wireless networks. Therefore, the protocols conceived must usefully use the finite energy resources. Designing to taking into consideration the consumption of energy becomes a major challenge for the establishment of a viable network. In this respect, use effective and efficient techniques using energy consumption is essential to achieve energy savings significant and prolong the life of the nodes and, consequently, that of the network. Ideally, a communication protocol for ad hoc networks should operate knots only when needed. Otherwise, all nodes must be in sleep mode. Moreover, when a call is established, no data is required. In addition, the traffic should be distributed equitably among the nodes to avoid congestion and premature death of the network. Unfortunately such an optimal configuration is not feasible since it requires knowledge Preliminary of the traffic generation model and the network topology. Efficiency can be defined as the maximization of the life of the network as long as possible. In-depth research efforts have been led for the design of efficient energy management protocols. Several works have shown that the network activity is very expensive in energy. The delivery and the reception consume a lot of energy. However, Communication skills are not the only ones that consume energy. The energy consumed by an ad hoc node can be categorized into two categories: energy not related to communication and energy related to communication. The energy linked to the communication can be classified in message processing energy, the energy of the transmission, reception energy and standby power. The energy consumed by a node is also a function of its network activities at level of the different layers. At the physical layer and data link level, the nodes consume energy primarily to ensure their connectivity to the network. For this purpose, the nodes remain on listening to the channel and exchange control messages. In terms of routing, the energy consumption is due to the participation of the nodes to the routing operation and to the processing of the control traffic defined by the protocols.

\section{LITERATURE REVIEW}

Transmission power has a direct impact on the life of the batteries and their capacity of the network in terms of actual traffic carried. In addition, the range of a node directly influences the zone of interference. Increase the range, also involves increasing the probability of interference, the increase collision rate, decreases the capacity of nodes to transmit. The control of power or topology consists of adapting the ranges and transmission powers of the nodes to ensure a minimum consumption of energy while keeping the connectivity of the network. It is a question of finding an optimal transmission range for the nodes of the network, being able to be common or not, allowing to optimize the energy consumed during communications [3,13]. Topology control aims to reduce the range of nodes, if possible, from where reduce interferences and collisions for better conservation of energy [14]. Several proposals exist to ensure the control of topology and power. The mechanism proposed in [15] allows to adjust the power of a node until it has a limited number of neighbors. This does not ensure in all cases the connectivity of the network. Often nodes can end up in separate batches with a few direct neighbors. The purpose of the COMPOW [16] protocol is to adjust the power of the nodes according to a common value. This power level is the minimum level to ensure the connectivity of the network. This protocol highlights the importance of bidirectional links since a direct destination can only respond to a source if its transmission power mission is at least equal to that of the source. As a result, ensure a common power allows ensuring bidirectional links. This protocol also aims at increasing the capacity of transmission of the network with the lowest level of energy or range while keeping the connectivity of the network. Ensure minimal node reach while maintaining network connectivity poses the problem of finding the best network coverage and control of partitioning. The article [17] proposes to calculate the load at digraph on the whole nodes of the network, whose topology and location of the nodes is known at one instant given, then to deduce the Delaunay triangulation which allows to link the nodes having neighboring cells. The Delaunay diagram ensures total node connectivity network based on short links ensuring a minimum scope most of the solutions proposed at this level define a sleep mode during which a node limits its network activity in order to conserve its energy. One of the mechanisms of reference for the conservation of energy in wireless networks is PSM (Power Saving Mode) proposed by the IEEE 802.11 standard. From the energy consumption point of view, a node can have three states. It can be in reception, dissipating some energy, in broadcast, dissipating another energy or in standby mode (passive listening of the channel) and in this case it also dissipates energy. The 802.11 standard defines the PS (Power Save) for conserve node energy in a wireless network with or without infrastructure. A station using PSM can see one of the following two states at a given time: - Awake: the station uses all its power to send and receive packets at any time. Doze: the station is unable to transmit or receive, it 
uses the minimum of its energy. If it has messages to send, it saves them locally. In 802.11, two modes of energy management are defined: - Active mode (AM): the station can receive packets at any time. She is in the awake state. Power save (PS): The station may be in the state Doze but must wake up and return to the awake state to receive certain control, synchronization messages and receive the saved packets. All nodes in the network must be alive at the same time to change the messages they will have saved during the doze period. As a result, 802.11 defines a synchronization function that is based on the exchange of a packet called Beacon at the beginning of each Beacon Interval period. This period starts with a sub period called ATIM Window during which only the control messages and announcements (ATIM messages: Announcement Traffic Indication Message) on messages saved must be exchanged between the nodes. At the end of ATIM Window, if a node has not announced any packages to send and has not received any announcements so it can enter in Doze state for the rest of Beacon Interval. If not, he must remain Awake to carry out the necessary transfers and synchronize with all the nodes of the network at that level.

\section{PROPOSED METHODOLOGY FOR LOAD} BALANCING IN MULTIPATH NETWORK

The criterion for the shortest path depends on the minimum hop distance between the source-destination pair depending on which path is chosen. This national criterion selects central node pathways as hops between many sourcedestination pairs. This is why the transmission of the center node is higher than other nodes are the reason that the shortest path between any two points always passes through the center and the shortest path chooses the shortest route. Thus, it can be established that the load may be in equilibrium, if relaxed, we come up with a strategy that can drive traffic to the nodes in the center and allow for participation and diversity of active nodes as a whole. The other path with the peripheral node promises relatively optimum hoop distance while not choosing the center nodes of the network One way to achieve this is by comparison the optimum unit is not an absolute unit of measurement but a hop distance that does not introduce unacceptable delays.

The participation of the center node is not ignored, but it is important to emphasize its role in providing the shortest path to many source-destination joints and therefore its mitigation is an obligation.

We have used certain parameters to compute the load balancing

1. Compute the Minimum load among all possible nodes in all node as next hop and

2. Relatively smaller hop count than the optimal path

The number of routes a node can support, the hop between the valid source-destination paths can be judged by the number of routes returned to the source. Intuitively, we can argue that if an RREP comes back, it does There is a high chance that the corresponding node will participate as an intermediate hop for data transfer. So, the conclusion is, the larger the RREPs return through the node, the greater the chance that it will be the center node of the network. Or, if not locally involved, we can comment on the neutral load distribution at that node which should be reduced.

\subsection{The load metric in Multipath network}

Each node maintains a counter-labeled load that will calculate all unique RREPs returned through it. Now, whenever a node returns an RREP, it will add to the RREP with its own load counter of the load field, and then return the RREP to the top node. This RREP counters in the RREP packet until it finally reaches the source. Source and intermediate nodes then choose among the valid RREPs that they adopt the best load balancing paths using the criteria mentioned above. Therefore, we have two novels for loads, yet they are different in the context of the node and the RREP, the former being an indication of the understanding of the node and the latter being a measure of the understanding of a path

\subsection{Criteria for choose path}

The first criterion is to help us find a route that is too low or in other words not too much traffic in transit. The additive value of the load featured in the RREP is the measurement of the load across all nodes below that node. The dynamics of the node can often change this value, but due to regular routing, the routing table ensures that the load is kept at a new value than the bus.

The second criterion is to ensure that we do not choose larger routes in the pursuit of load balances that can lead to greater delays. Therefore, we choose the routes that are relatively favorable. Optimal optimization can only be achieved with the approval of the next hops that are separated from the optimal only by a recognized number of hops. The acceptable number of hops is dependent on the network diameter and this is another area of study but in our approach the maximum hop difference was fixed to 4 , which is an experimental value with no good results beyond that.

The main idea of Multipath Protocol is to calculate multiple paths within the root discovery method to prevent link failure. In fact, the main purpose of conceptualizing this protocol is to search multiple routes during the same route discovery process, but only the best path is selected based on a few metrics (number of hops) and is used for data transmission between source and destination. If the primary path fails, the other path is used only.

\subsection{Step for proposed algorithm}

- If an RREP is received, the only route format from the source is used to send the data packet.

- If many RREPs are accepted, the source chooses the best route according to the short number of "hop counts". Other routes are awaiting RERR packets that indicate failure of the root route; In this case the best path from the alternative path is used to transmit information. The routing decisions are as follows:

If (no path is available)

\{

Call path discovery process:

path_discovery(source, Destination, Hop_Count )

\}

If (1==path_discovery(source, Destination, Hop_Count )) \{

Number of path $==1$

Then forward the data packet without load balancing to the discovered route

\} path $(\mathrm{N})>1)$

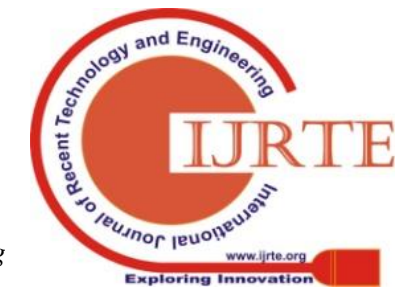


Then call load_balancing(N) method to discover best optimal path

\}

\subsection{New proposed metric}

Selects routes with the lower hopcount to forward the protocol information. However, lower compression routes may provide shorter delays than the route providing lower hop count. To select low compression routes, we need a new metric that allows the source node to select low congestion routes. Because of this, we propose according to Equation (1) the following is a new metric for achieving load balancing among selected routes considering the number of active paths through each node:

$$
\operatorname{Min}\left[\frac{1}{n_{p}} \sum_{i=1}^{n_{p}}(\text { sizeof }(\text { buffer }(i))\right.
$$

Where buffer(i) means the size of the buffer occupation of the link i transfers to an intermediate node participating in route $\mathrm{p}$. With hops count, Route $\mathrm{P}$ which ensures that the metric takes the hop count number into account to estimate the traffic load.

Maximum_buffer_size is defined as the maximum occupation size of the buffer within each intermediate node. Occupation buffer size for each intermediate node (buffer_size i) is always less than or equal to max buffer_size i.

In such protocols, link failure of the primary pathway, through which the transmission of information actually takes place, can switch the source to the alternate path rather than to discover another source. Discovering a new route occurs only when all precomputed paths break.

To develop a new protocol we have used the modified header of RERP and RERQ packet structure to transmit those packet in the route to compute the optimize path and for computation of optimized path and save the path information in packet we added a new field in the RREP packet structure which is buffer size which store the information of every route discover by the routing protocol and it maintain the information about their source and destination node of current path. It divides the value of the buffer size field by the expectation of each route between the source and the destination for the compression level.

\begin{tabular}{|c|c|c|c|c|c|}
\hline $\begin{array}{l}\text { Sour } \\
\text { ce } \\
\text { Nod } \\
\text { e }(S)\end{array}$ & $\begin{array}{l}\text { Destinat } \\
\text { ion } \\
\text { Node } \\
\text { (D) }\end{array}$ & $\begin{array}{l}\text { Sequence } \\
\text { Number } \\
\text { (Seq_Num } \\
\text { ber) }\end{array}$ & $\begin{array}{l}\text { Hop_co } \\
\text { unt }\end{array}$ & $\begin{array}{l}\text { Time } \\
\text { out }\end{array}$ & $\begin{array}{l}\text { Buffer } \\
\text { Size }\end{array}$ \\
\hline
\end{tabular}

The algorithm for calculating the compression level of each route between source and destination is as follows:

\section{Variables Used In load balancing:}

$\mathrm{N}=$ Number of routes

$\mathrm{P}=$ Packet to be sent

$\mathrm{TB}=$ Block size to be sent using load balancing

Rolling $=$ Boolean variable indicating whether the process has already started load balancing

$\Sigma \mathrm{Ai}=$ Sum of contributions from all routes

Arrangements:

- The arrangement A(i) must be ordered ascending, and others Arrangements must be arranged parallel to $\mathrm{A}(\mathrm{i})$

- $\quad$ BW (i) = bandwidth of each route

- $\quad$ L (i) = maximum capacity available for use channel flow before initiating load balancing
- $\quad \mathrm{CB}$ (i) = Amount sent by the route $\mathrm{i}$ using load balancing (i) the contribution of each route

- $\mathrm{Mq}=$ Message Queue

Subroutines:

- Send (i, p) is the function that sends the packet through the channel Pi. Does not return results.

- Next (): This function returns the next packet to be sent, or the algorithm keeps waiting until it is created.

- $\quad$ Tam (p): Returns the size of a packet p.

- Util (i): Returns the number of channel reservation currently used by the flow path $i$.

\section{Algorithm Load Balancing; \\ Call Load_balancing (N)}

$$
\mathrm{p}=\operatorname{Next}()
$$

existing_path(True) $\& \& i \leftarrow 0$

Make

$$
\mathrm{i} \leftarrow \mathrm{i}+1
$$

$$
\begin{aligned}
& \text { Until (i> N) } \|(\text { Util (i) }<\text { L (i)) } \\
& \text { Yes (i> N) } \\
& \quad \text { Send (i, p) } \\
& \text { p = Next }() \\
& \text { If not }
\end{aligned}
$$

$$
\begin{aligned}
& \mathrm{i} \leftarrow 1 \\
& \text { For } \mathrm{i}=1 \text { to } \mathrm{N} \\
& \text { CB (i) } \leftarrow 0 \\
& \mathrm{Mq}(\mathrm{i}<\mathrm{N}) \\
& \text { Send (i, p) } \\
& \mathrm{CB}(\mathrm{i})=\mathrm{CB}(\mathrm{i})+\operatorname{Tam}(\mathrm{p}) \\
& \text { If }(\mathrm{CB} \text { (i) }>\mathrm{TB} \| \mathrm{CB} \text { (i) }>\Sigma \mathrm{Ai}) \\
& \text { Congession_level=sizeof(buffer(i)/hop_c } \\
& \mathrm{p}=\text { Next }() \\
& \text { ount); } \\
& \mathrm{i} \leftarrow \mathrm{i}+1
\end{aligned}
$$

End Load_balancing procedure end Algorithm

\section{RESULT ANALYSIS}

We use NS2 to simulate our proposed protocol. The following parameters are selected for the initial simulation and system validation:

TABLE II. PARAMETERS OF SIMULATION

\begin{tabular}{|l|l|}
\hline Parameter & Value \\
\hline Dimensions & $1000 \times 1000 \mathrm{~m}^{2}$ \\
\hline Number of nodes & 30 \\
\hline Simulation time & $300 \mathrm{~s}$ \\
\hline Source type & $\mathrm{CBR}$ \\
\hline Number of Connections & 10 \\
\hline Packet size & 512 bytes \\
\hline MAC Layer & IEEE $802.11 \mathrm{~b}$ \\
\hline Buffer size & 50 packets \\
\hline Propagation Radio Model & Two Ray Ground \\
\hline Physique Layer & Band Width as $2 \mathrm{Mb} / \mathrm{s}$ \\
\hline Maximal speed & $10 \mathrm{~m} / \mathrm{s}$ \\
\hline Pause time & $10 \mathrm{~s}$ \\
\hline Interval time to send & $2 \mathrm{packets} / \mathrm{s}$ \\
\hline
\end{tabular}

All nodes have the same transmission range of 200 meters. The selected mobility model is the random waypoint model. In this mobility model, a node

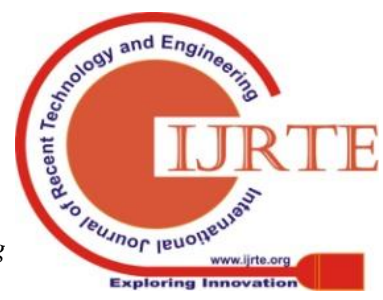


moves towards the destination with the minimum speed and the selected speed at the same speed at the same time.

\subsection{Evaluation of Proposed Protocol (NCLB-AOMDV)}

We compare the result of proposed NCLB- AOMDV protocol to evaluate the performance of different parameters. We have compare the result of LB-AOMDV, SMR \& AOMDV routing protocol with proposed protocol. We study the variation effect of the following metrics:

- Packet Delivery Ratio (PDR).

- $\quad$ Average end to end packet delay (APD).

- Energy Spent

\subsection{Simulation results}

We have successful implemented the proposed protocol and achieve the result

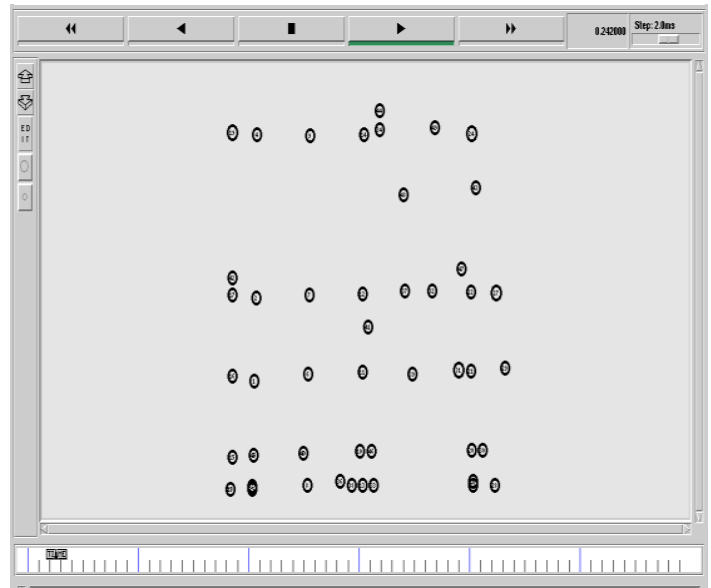

Fig1 : Distribustion of node in Wireless network

Above figure show that when we apply our proposed algorithm to exiting routing scheme then number of path is reduce and it show only optimized path available between the network

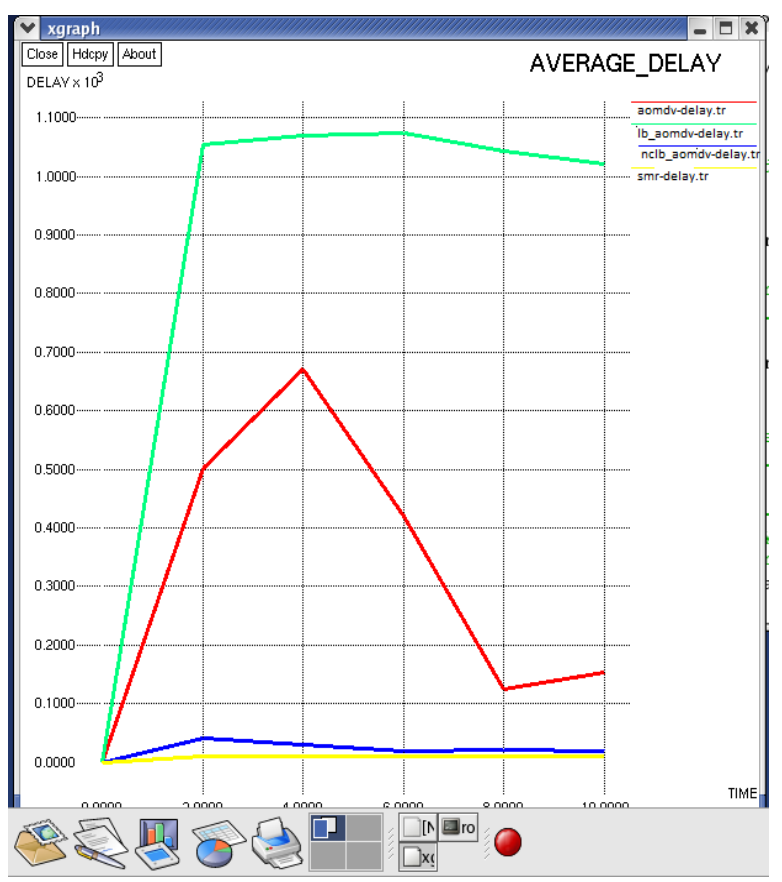

Fig2: Comparative analysis of Average delay in multipath Network

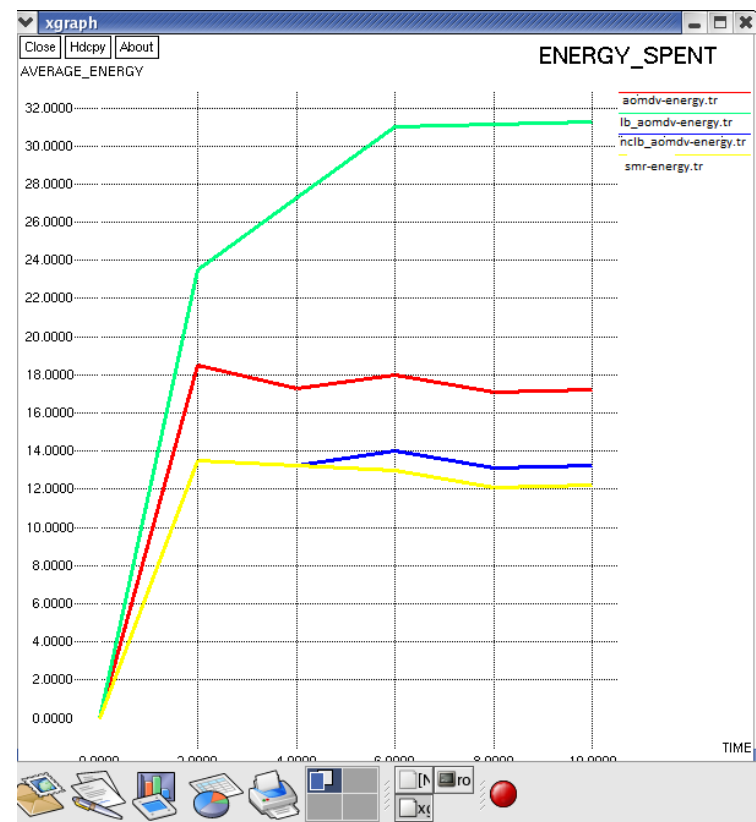

Fig3: Comparative analysis of Average energy spent in multipath Network

In proposed protocol result show that if the number of node is increased i.e. number of connection is increased then network traffic load is decreased up to 40 establish connection. which show that load is distribute to different route when the traffic is increased it consume less the $60 \%$ less energy to transmit the packet compare to LB-AODMV protocol and $50 \%$ less energy than AOMDV

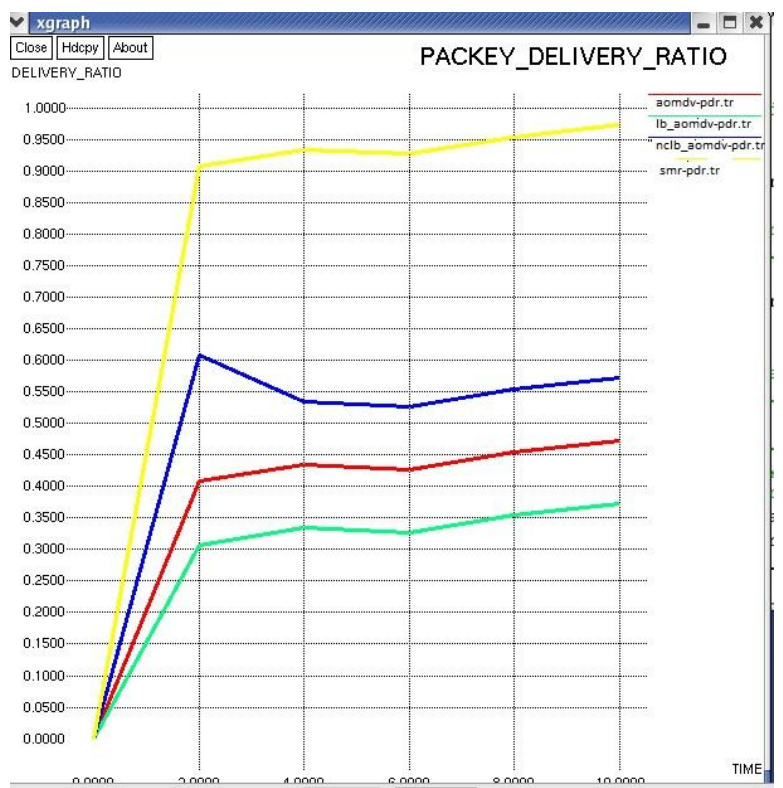

Fig 4 : Comparative analysis of packet deliver ratio of multipath network

Fig 4 shows that the packet delivery ratio decreases according to connection number. Multipath routing performance is predictable under heavy loads. When the traffic load is about 40 connections (which is a heavy load), the PDR obtained by the LB-AOMDV protocol is $9 \%$ better than the PDR obtained by our proposed NCLB-AOMDV protocol, still the new protocol has better PDR than that of SMR \& AOMDV protocol.

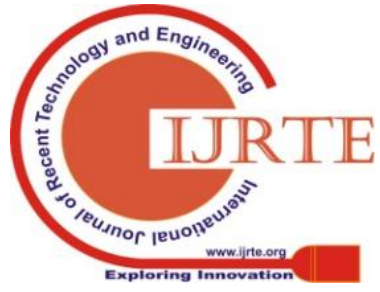


After computing the all parameters our proposed protocol work good when the number of node is greater than 40 node, when the network connectivity is high then the performance of NCLB-AOMDV protocol is better in terms of Delay \& Energy spent and is second best in terms of Packet Delivery rate.

\section{CONCLUSION}

In this paper we have analysis the load balancing in Multipath routing to achieve better network performance. We have study and reviewed the different load balancing algorithm in multipath network and based on review we have proposed a new load balancing algorithm in the multipath network. We have modified the packet of RREP and RREQ packet to transmit and receive the route information to compute the load of every path and we added a new field in RREP packet Buffer size to store the information of the current node and their neighbors, inorder to find the alternate route if network load, in increase we have compare the performance of proposed protocol with the LB-AOMDV, AOMDV \& SMR multipath routing protocol. Proposed routing protocol performance show that if network load is increase the load is distributed to the different route and overall load of current route is decrease, and as the objective was to achieve lesser delay by providing load balancing based on Contribution of each existing route.

\section{REFERENCES}

1. M. A. Feki, F. Kawsar, M. Boussard, and L. Trappeniers, "The internet of things: the next technological revolution," Computer, vol. 46, no. 2, pp. 24-25, 2013.

2. J. Yick, B. Mukherjee, and D. Ghosal, "Wireless sensor network survey," Computer Networks, vol. 52, no. 12, pp. 2292-2330, 2008.

3. L. D. Xu, W. He, and S. Li, "Internet of things in industries: a survey," IEEE Transactions on Industrial Informatics, vol. 10, no. 4, pp. 2233-2243, 2014.

4. Y.-W. Wang, H.-L. Yu, and Y. Li, "Internet of things technology applied in medical information," in Proceedings of the IEEE International Conference on Consumer Electronics, Communications and Networks (CECNet '11), pp. 430-433, April 2011.

5. G. Chong, L. Zhihao, and Y. Yifeng, "The research and implement of smart home system based on internet of things," in Proceedings of the International Conference on Electronics, Communications and Control (ICECC '11), pp. 2944-2947, IEEE, Zhejiang, China, September 2011.

6. P. Vlacheas, R. Giaffreda, V. Stavroulaki et al., "Enabling smart cities through a cognitive management framework for the internet of things," IEEE Communications Magazine, vol. 51, no. 6, pp. 102111, 2013.

7. Zigbee Alliance, ZigBee Specification Version 1.0, 2004, http://www.zigbee.org.

8. C. Perkins, E. Belding-Royer, and S. R. Das, "Ad hoc on-demand distance vector (AODV) routing," IEEE RFC 3561, 2003.

9. M. K. Marina and S. R. Das, "Ad hoc on-demand multipath distance vector routing," Wireless Communications and Mobile Computing, vol. 6, no. 7, pp. 969-988, 2006.

10. M. Hammoudeh, O. Aldabbas, S. Mount, S. Abuzor, M. Alfawair, and S. Alratrout, "Algorithmic construction of optimal and load balanced clusters in wireless sensor networks," in Proceedings of the 7th International Multi-Conference on Systems Signals and Devices (SSD '10), pp. 1-5, IEEE, Amman, Jordan, 2003.

11. J. Zhang and T. Yang, "Clustering model based on node local density load balancing of wireless sensor network," in Proceedings of the 4th International Conference on Emerging Intelligent Data and Web Technologies (EIDWT '13), pp. 273-276, Xi'an, China, September 2013.

12. Y. Liao, H. Qi, and W. Li, "Load-balanced clustering algorithm with distributed self-organization for wireless sensor networks," IEEE Sensors Journal, vol. 13, no. 5, pp. 1498-1506, 2013.
13. Y. Wu and W. Liu, "Routing protocol based on genetic algorithm for energy harvesting-wireless sensor networks," IET Wireless Sensor Systems, vol. 3, no. 2, pp. 112-118, 2013.

14. A. Bhatia and P. Kaushik, "A cluster based minimum battery cost AODV routing using multipath route for ZigBee," in Proceedings of the IEEE 16th International Conference on Networks (ICON '08), pp. 1-7, New Delhi, India, December 2008.

15. K. Kim, K. Cho, and S. Bahk, "Neighbor-aware adaptive load balancing algorithm for dense wireless sensor networks," in Proceedings of the IEEE VTS Asia Pacific Wireless Communications Symposium (APWCS '09), 2009.

16. J. M. Lucas and M. S. Saccucci, "Exponentially weighted moving average control schemes: properties and enhancements," Technometrics, vol. 32, no. 1, pp. 1-12, 1990.

17. D. Satyanarayana and S. V. Rao, "Constrained Delaunay Triangulation for Ad Hoc Networks," Journal of Computer Systems, Networks, and Communications, vol. 2008, Article ID 160453, 10 pages, 2008. https://doi.org/10.1155/2008/160453. 